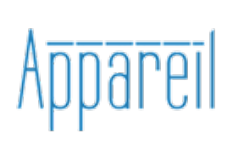

Appareil

13 | 2014

Trauma et création

\title{
Entre le trauma et le coma
}

Entretien avec Germain Rœsz

\section{Jean-Louis Déotte}

\section{(2) OpenEdition}

Journals

Édition électronique

URL : http://journals.openedition.org/appareil/2002

DOI : 10.4000/appareil.2002

ISSN : 2101-0714

Éditeur

MSH Paris Nord

Référence électronique

Jean-Louis Déotte, «Entre le trauma et le coma », Appareil [En ligne], 13 | 2014, mis en ligne le 23 juillet 2014, consulté le 30 juillet 2020. URL : http://journals.openedition.org/appareil/2002 ; DOI : https:// doi.org/10.4000/appareil.2002

Ce document a été généré automatiquement le 30 juillet 2020.

\section{(c)}

Appareil est mis à disposition selon les termes de la Licence Creative Commons Attribution - Pas d'Utilisation Commerciale - Pas de Modification 4.0 International. 


\title{
Entre le trauma et le coma
}

\author{
Entretien avec Germain Rœsz
}

Jean-Louis Déotte

1 La question traitée sera celle des rapports entre les arts, ici la peinture, et les effondrements psychiques, individuels ou collectifs. Elle partira du constat qu'il y a de telles discontinuités historico-politiques que l'hypothèse métaphysique d'une "raison dans l'histoire" est bien problématique, parce que l'humanité n'est pas un sujet collectif, lequel pourrait se ressaisir, se réfléchir au moins comme «esprit » dans sa totalité depuis un supposé commencement. Quand il y a eu des survivants pour tel ou tel génocide, qu'ils puissent et doivent témoigner, c'est nécessaire, mais cela ne permettra pas réellement de sortir de «l'époque de la disparition» ouverte avec le génocide des Arméniens en 1915. Que nous soyons toujours dans une telle époque, le succès des séries télévisuelles consacrées aux fantômes, aux morts-vivants et autres spectres nous en fournit constamment la preuve. De même l'importance de ce thème central chez Derrida. Nous sommes même les stricts contemporains d'une série de guerres génocidaires qui a ravagé l'Afrique des Grands Lacs au cours des années 1997-2002, dont l'épisode le plus connu est le génocide rwandais, génocide qui déclencha une succession de massacres pour lesquels il n'y eut pas de témoins selon les historiens eux-mêmes ${ }^{1}$. La guerre se rallume régulièrement dans cette région, elle peut même être provoquée par ceux qui ont été des victimes.

Voilà qui conduirait à mettre à l'épreuve la thèse de Warburg et Didi-Huberman, celle d'une survivance des formes esthétiques. Pour qu'il y ait mémoire (individuelle ou collective), il faut bien un support continu ${ }^{2}$. Et pour les arts, cela suppose l'existence du musée, qui n'est qu'une invention du xvIII siècle. Et encore faut-il qu'il y ait des lecteurs pour ces souvenirs de catastrophe. Les archéologues ont découvert au Japon après le dernier tsunami que des stèles très anciennes en pierre dans la montagne audessus de la côte ravagée témoignaient de la dernière montée des eaux et précisaient qu'il ne fallait pas construire en-deçà. Mais eux seuls savaient encore les lire. Comment signalera-t-on pour des êtres raisonnables dans quelques milliers d'années qu'ici ou là il y a des dépôts de déchets nucléaires ultimes (intraitables) ? Ces questions, Platon se les pose au début du Timée, rapportant les dires des prêtres égyptiens : l'Égypte aurait 
été maintes fois effacée de la surface terrestre. Et c'était pourtant une civilisation de l'écriture! On peut faire l'hypothèse que c'est à partir de là qu'il développera sa critique de l'écriture comme oublieuse mémoire (le Phèdre) et sa philosophie de l'anamnèse parce que l'âme immortelle a toujours déjà tout appris (le Ménon).

$3 \quad$ L'enfant Useppe de La Storia d'Elsa Morante (1974-1977) 3 puise dans un double fonds sensoriel : d'une part les photos prises après l'ouverture des camps nazis, qu'il aperçoit sur une place romaine dans la devanture d'un kiosque à journaux, d'autre part, les sensations de son corps renaissant après une crise d'épilepsie. Les premières sont pour lui strictement inintelligibles (amas de cadavres, corps pendus à des crocs de boucher, etc.). Ne les comprenant pas, il ne les identifie pas ; à proprement parler, il ne les voit même pas, il n'en garde aucun souvenir conscient. Dans un premier temps, ces images provoquent un choc sans écho. En outre, elles ne font pas partie de ces traces mnésiques qui, paradoxalement, ne seraient pas inscrites. Ce qui redouble leur effroyable charge émotive. Elles appartiennent à l'immémorial historique comme tout ce qui, selon Benjamin, est perçu dans le cadre d'une «esthétique du choc » par une perception de distraction, essentiellement non focalisante, comme celle provoquée par l'architecture ou le cinéma. Ainsi, à partir de cette rencontre fortuite, les facultés langagières d'Useppe, déjà loin d'être vraiment matures, se dégradent. Ses bizarreries mentales, qui avaient déjà alerté les médecins et psychiatres, s'« organisent», se "systématisent » en un syndrome, il tombe en proie au haut-mal, auquel il finira par succomber après une succession de crises toujours plus graves et plus rapprochées. Chaque chute coïncide avec une perte totale de conscience, c'est l'expérience d'une disparition onirique, d'un blanc, sans le souvenir des images du rêve. Il ne s'agit donc pas de souvenirs inconscients, mais d'un immémorial pur, d'une déconnexion momentanée de tout l'appareil psychique qui peut affecter chacun d'entre nous pris, par exemple, dans une absorption attentive maximale. C'est le cas de ces enfants dont on dit qu'ils sont dans la lune.

4 Puis, chez lui, après la crise qui ne dure que quelques instants, le corps peu à peu reprend ses droits, jusqu'à un état de quasi béatitude, de bonheur purement physique, avant de sombrer dans un profond et durable sommeil sans reste.

5 La Storia encadre l'histoire de quelques individus, d'une mère et de ses deux fils (et leurs amis, parmi lesquels les chiens ont une place essentielle) par la "grande histoire ", dont les événements résumés aux découvertes technoscientifiques (l'atome), aux combats politiques et militaires, entre prise de pouvoir des fascistes et Guerre froide, ouvrent chaque chapitre. La succession des révolutions et de leurs répressions est telle qu'il est vain de concevoir une quelconque raison à l'œuvre dans l'histoire. Morante n'est ni hégélienne ni marxiste, partageant avec Pasolini une même haine du pouvoir. Une seule chose semble avérée, c'est que «l'histoire continue» comme un fleuve de sang est, elle aussi, la toile de fond du peintre G. Rœsz, ancien apprenti boucher des abattoirs de Colmar en Alsace.

Il n'y aura donc pas de «Tribunal de l'histoire » censé, au bout du compte, asséner à l'humanité lucide quelques leçons désormais inoubliables, du type: «Plus jamais cela!» D'autant que, pour Morante, les survivants des camps comme ceux des «expériences» nucléaires réalisées au Japon par les Étatsuniens ne porteront pas témoignages. Ils en auraient certes la possibilité, ce n'est pas le problème de l'irreprésentabilité ou de l'indicible, mais de l'absence de témoins intéressés. Rappelons que Le Différend de Lyotard ${ }^{4}$ s'ouvre par une analyse identique. 
Morante :

[Les survivants des camps, en particulier les quelques Juifs de retour à Rome] apprirent vite que personne ne voulait écouter leurs récits : certains cessaient de les écouter dès les premiers mots, d'autres les interrompaient rapidement sous un prétexte quelconque, et d'autres encore les écartaient carrément en ricanant, comme pour leur dire : «Frère, je te plains, mais en ce moment, j'ai autre chose à faire. "

De fait, les récits des Juifs ne ressemblaient pas à ceux des capitaines de navire ou d'Ulysse, le héros, de retour dans son palais. Ils étaient des figures aussi spectrales que des nombres négatifs, en dessous de toute vision naturelle et incapables de susciter même la plus banale sympathie. Les gens voulaient les éliminer de leurs journées, comme dans les familles normales on élimine la présence des fous ou des morts. Et ainsi, en même temps que les figures illisibles qui fourmillaient dans leurs noires orbites, de nombreuses voix accompagnaient les petites promenades solitaires des Juifs, se répercutant, énormes, dans leurs cerveaux en une fuite en spirale, plus bas que le seuil habituel de l'audible. ${ }^{5}$

8 Leur situation, inintelligible pour une esthétique et une politique comme celle de Rancière, est celle d'un tort absolu parce que leurs interlocuteurs sont comme l'enfant de la Storia. Ses comas répétés sont les nôtres sur la scène de l'histoire. C'est le choc nerveux qui provoque le coma, lequel ne saurait être alors considéré comme le moment du négatif dans une quelconque dialectique ou odyssée de l'esprit. Dans la vie des individus comme dans celle des sociétés, il y a des blancs comme dans la physique de Descartes où Dieu recrée l'univers instant après instant, toujours à nouveaux frais, puisque sa volonté est infinie et qu'il pourrait créer un état du monde absolument inouii. On retrouve cette fiction chez Lyotard lui-même avec sa théorie de la phrase, du qu'arrive-t-il ?, du quod ? qui est un monde à elle seule, absolument détachée de tout, en particulier des autres phrases qui surgissent de-ci de-là, comme en tombant. Le Différend introduit un coma entre chaque phrase ou état du monde, ce n'est que dans un second temps que la question de l'enchaînement d'une phrase sur l'autre se pose. L'humanité est globalement épileptique.

On pourrait déduire du constat benjaminien de la " fin de l'expérience $»^{6}$, qui est due à la fin de la narration et donc de la tradition, la même certitude, celle d'un coma universel, à condition d'introduire une autre dimension: celle de la destruction des repères géographiques (et finalement chtoniens) sans lesquels il n'y a pas de structuration narrative en raison du bouleversement total du paysage, guerrier ou urbain. Verdun et Alexanderplatz. Ce qui croitt alors, à la place d'un territoire quadrillé par des points-clef ${ }^{7}$, c'est la zone, au sens de Tarkovsky (Stalker) ${ }^{8}$. La zone, comme le montre D. Skopin, n'est pas un non-lieu, car quelque chose nous y attend. Y a-t-il pour autant un anthropomorphisme de la zone ? Une sorte d'inquiétante étrangeté ?

$\mathrm{Au}$ fond, notre conception du témoignage était optimiste : certes, disions-nous avec Derrida et Lyotard, un témoin n'est jamais à la hauteur de ce qu'il a vécu, car quand l'événement a eu lieu, le témoin ne pouvait l'appréhender comme tel sinon ce n'aurait pas été un événement, par définition inouï, mais une sorte d'anomalie statistique ou de hasard accidentel, comme la chute inopinée d'une tuile sur la tête d'un citadin qui eut le tort ce jour-là de passer dans cette rue-là. Le témoin n'est jamais dans l'immédiateté de l'événement, ou dans ce qu'on appelle aujourd'hui « le temps réel » : il lui faut donc se constituer progressivement comme support d'inscription de ce que l'on avait encore jamais vu. Mais quand il y parvient il y a bien longtemps que l'événement est passé, du passé. La théorie freudienne de l'après-coup désarmait la prétention de pouvoir être le 
témoin d'un véritable événement et rendait légitime une écriture de l'expérience de l'événement grâce au retard. Cela suppose quand même un sujet plus ou moins doué de conscience, la continuité de fonctionnement d'un appareil psychique ou de la conscience phénoménologique (Husserl).

11 Tout autre est la réalité du coma : l'épileptique sombre dans une nuit totale, le temps nécessaire à l'inscription des traces psychiques n'existe pas. L'épileptique disparaît à lui-même. Il n'y a que des discontinuités sans patrimoine obscur.

Où se logerait alors, en dehors du musée universel, la possibilité des survivances immémoriales censées peupler l'imaginaire des hommes et des artistes en particulier? Est-ce que ce n'est pas une douce fantasmagorie, celle de la permanence de formes privilégiées constituant la culture depuis les origines (Warburg)?

Le monde de l'épileptique, tout en discontinuités, est à l'opposé de celui de l'hystérique qui peut être figé dans une posture par l'hypnose ${ }^{9}$ et la photographie ${ }^{10}$.

Dès lors, les ressources de l'artiste ne sont pas du côté de la survivance des formes, mais des processus physiologiques et en particulier des humeurs. Lesquels sont, d'après la leçon de Benjamin et Kracauer, la grande affaire du cinéma ${ }^{11}$.

Dès lors, la peinture de Rœsz ${ }^{12}$ suppose l'appareillage cinématographique de la sensibilité commune: les flux cinématographiques privilégient certes le mouvement (poursuites, road movies, etc.), mais s'imposent picturalement par des extraits verticaux de bandes passantes accrochées là attendant un hypothétique montage. Ce sont ses Linéales comme autant de séquences ou de phrases-images. Certaines sont redoublées par des découpes encéphalogrammatiques de métal oxycoupé au tracé presque plat. Rœsz, jeune adulte, est revenu au monde à partir d'un long coma provoqué par un accident de la route qui aurait dû être son tombeau. C'est la très longue hospitalisation qui fut fondatrice, parce qu'elle interrompit durablement toute activité :

L'origine de ma peinture se situe dans un choc fondateur, un trauma comatique. $\mathrm{Ne}$ pas voir ni penser le monde. Savoir, au retour, parmi les vivants et la parole qu'une partie de soi-même, de sa conscience fut abolie, oubliée. [...] Mais cette anecdote qui est la mienne comment peut-elle interroger celui qui regarde? Comment et pourquoi serait-il concerné ? $^{13}$

17 Virilio donne une réponse dans son Esthétique de la disparition ${ }^{14}$ : ces états, qui ne sont pas nécessairement liés à un trauma, sont très fréquents, très fugaces et pour cette raison inaperçus chez chacun.

Rœsz n'était pas destiné à devenir artiste (qui l'est d'ailleurs en dehors de ceux qu'une loi commande un jour de peindre, d'écrire, etc. ?). Il faut souvent l'interruption de la vie par la maladie (Matisse), l'accident (l'avion de Sam Francis s'écrasant dans le désert), l'incarcération (Stiegler), l'isolement (Descartes), la solitude (Woolf) pour venir briser l'élan vital. Car sans cela, la répétition de l'habitus social, de l'hexis corporel s'imposeraient à tous, toujours. Et il n'y aurait pas d'art.

Dès lors, chez ce grand comateux que fut Rœsz, une évidence capitale s'imposa : les signes n'ont pas tellement d'importance, car il n'y a pas eu initialement d'inscription, faute de surface d'écriture ou de bloc magique, même si ça et là des phrases apparaissent, voire des sortes de proto-écritures ${ }^{15}$. Même s'il veut, comme tout un chacun, faire traces. Ses peintures sont plutôt des coulées de couleur prises dans le grand fleuve sans mémoire (Léthé). Cette peinture échappe donc à la Grande Sémiologie qui s'impose à l'art et à sa théorie depuis qu'il y a des empreintes, des marques, des 
incisions, des traces et autres masques mortuaires. Ce n'est pas un univers de formes et de figures parce que le médium de la peinture est liquide. Arcs-en-ciel, décalcomanies, irisations de bulles de savon, papiers buvard, dilution par quoi le recto et le verso de la toile se confondent, pliages et dépliages aléatoires, peintures blanches les plus translucides possibles. Bref, tout l'univers mouillé de la première esthétique de Benjamin ${ }^{16}$. Comme le rappelait Benjamin, l'humanité vit au fond d'un grand océan vertical, parce que l'atmosphère, c'est de la vapeur d'eau plus ou moins dense. Bref, à la différence de son ami et complice Gagean, peu de matériologie chez Rœsz, dont la ville (Colmar), celle du retable d'Issenheim de Grünewald, est bâtie sur les eaux de l'Ill et de ses affluents qui envahissent régulièrement les caves des maisons. Rœsz qui privilégie les promenades au bord des fleuves, si possible, en crue, donc sans limites et sans frontières :

Mon goût des irisations, au début de ce que j'appelle ma peinture. Grandes surfaces traversées de signes (écrits) blancs colorés, frémissements de la couleur. Blanche(s) en était le titre. [...] Mais l'eau n'est pas à représenter, elle se déplace dans le projet de faire peinture, d'écarter le réel pour le réel de la peinture. ${ }^{17}$

Que la couleur s'émancipe de la forme et de la figure, qui sont plutôt en rapport avec le langage, avec la loi, cela l'enfant le sait bien, l'enfant qui, surpris par l'adulte, sent malgré lui que le rouge lui monte aux joues. L'affect coloré envahit le corps et se répand d'autant plus dans les zones où la singularité apparaît aux autres. Il peut y avoir un système des couleurs si on leur donne des noms, on peut même déconstruire ces systèmes qui après tout sont des organisations culturelles, et donc historiquement et culturellement datées, mais comment déconstruire des couleurs sans contours, sans frontières, passant de l'une à l'autre, au contraire d'un Adami ?

Horizon

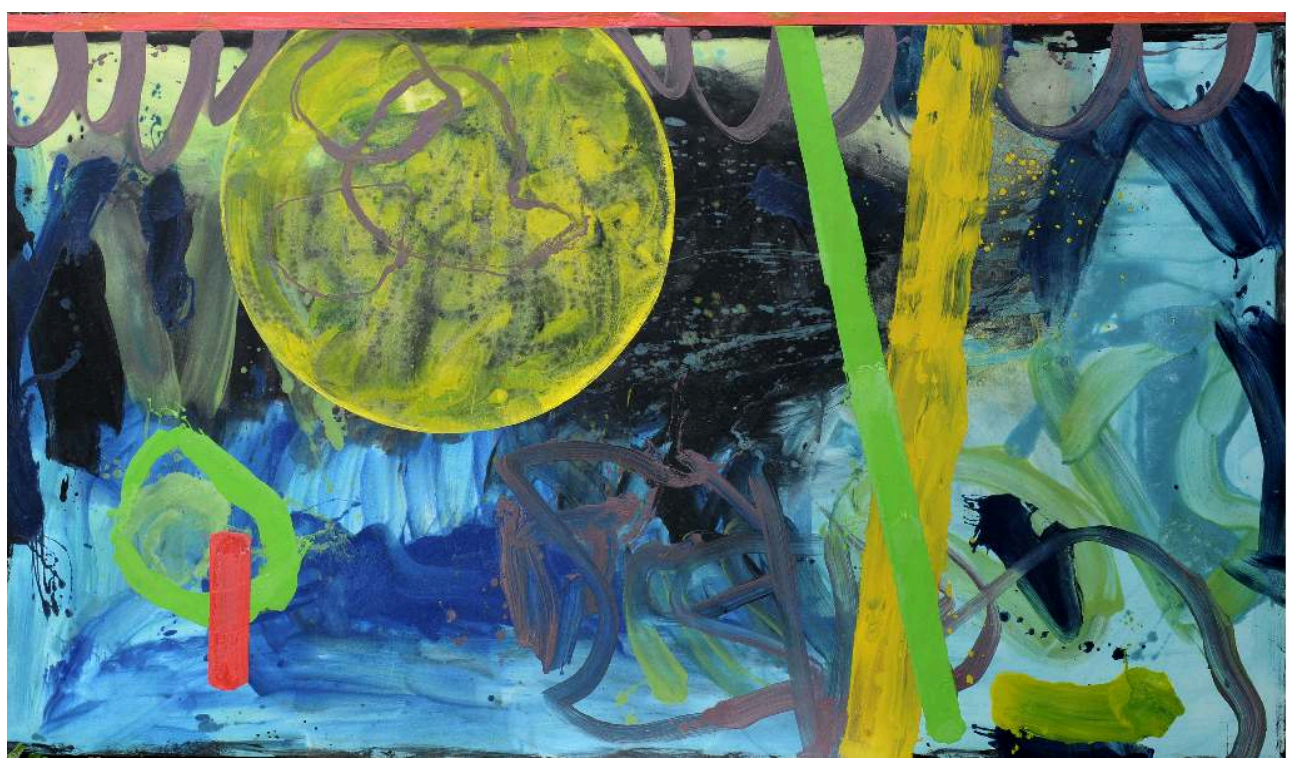

2003-2004

Photo P. Bouvier

21 Sans en avoir l'air, c'est donc toute une pensée (poststructuraliste) de l'espacement comme condition de la pensée comme de l'existence singulière ou communautaire (Nancy), qui est mise à mal. Le sol qui devient une nappe, n'est plus le plan d'inscription de l'ordre symbolique, ce que privilégiait selon Benjamin le dessin, mais l'étal d'une 
peau qu'on plie et déplie comme chez Franju (le cinéaste du Sang des bêtes mais aussi des Yeux sans visage... et donc des greffes de peau).

Peindre, dans la chambre, dans la salle de réunion de la coopérative des bouchers. Dans le lieu de séchage des boyaux. Je ne tenais pas debout dans ce lieu. J'étais, tout le temps, penché sur la toile. Ce rapport au sol est toujours une constante dans l'élaboration de ma peinture (cela rencontre bien entendu les procédures de Pollock, mais aussi de Motherwell). ${ }^{18}$

À une époque où les artistes exploitent le plus loin possible les potentialités du virtuel (la digitalisation), c'est-à-dire se font toujours davantage les serfs du langage, de la communication, même si ce langage ne laisse place à aucun locuteur (les constituants d'une image numérique ne sont que des suites de chiffres), Rœsz non seulement n'effectue aucun retour à la projection classique inaugurée à la Renaissance (stade ultime : Le Grand Verre par Marcel Duchamp ?), mais plus largement ignore ce qui en était encore la condition: l'inscription. Car il n'y a pas de tableau comme fenêtre ouverte sur le monde sans tracés de ce qui apparaît là-bas, derrière le plan de projection. Ce qui implique que tout un langage critique est invalidé, de l'archi-trace à l'empreinte.

23 Si le medium (l'eau), c'est le message (le pigment), inversement, le message (l'affect) contamine le medium (la peau). Il y a une essentielle porosité entre les deux. La porosité, une notion essentielle dans la pensée de Benjamin, n'est pas seulement une qualité de la matière pierreuse, un état de la lave solidifiée. On sait depuis le Journal de Naples de Benjamin et Lacis qu'elle est l'opérateur d'échange pour penser le rapport entre la forme et le contenu, entre le medium et le message. Il est inévitable alors que le peintre et sa toile coexistent ${ }^{19}$ dans la même Raum (le terme est de Rœsz) qui n'est pas l'espace de l'objectivation que considèrent le critique et l'historien de l'art, de la même manière que l'enfant Walter ne se cachait pas derrière les meubles de l'appartement de ses parents, mais faisant tout un avec eux, disparaissait à ses propres yeux ${ }^{20}$ croyant ainsi se faire oublier.

24 Chaque toile est ainsi une zone dans laquelle les cercles, les fragments sombres de fleuve, ne doivent pas être pris comme des formes-figures mais comme des contractures du corps qui se ressaisit peu à peu après la crise comatique, qui se déplie, ou les fractures et les organes éclatés. Ce qui remonte alors du passé, c'est le paysage du corps. Ce pourrait être le sens de la " phrase-affect » chez Lyotard" ${ }^{21}$.

Dans la pratique picturale de Rœsz, cet opérateur va se décliner en autant d'opérations secondaires, le pli étant le dernier en date puisqu'il permet le recouvrement de deux surfaces et l'absorption du pigment de l'une par l'autre. "Plier, c'est dessiner », dit-il. Dès lors le travail du peintre est sélectif : constater si une greffe de couleur a pris ou non. C'est une peinture réparatrice, à l'opposé de l'actuelle ingénierie. 


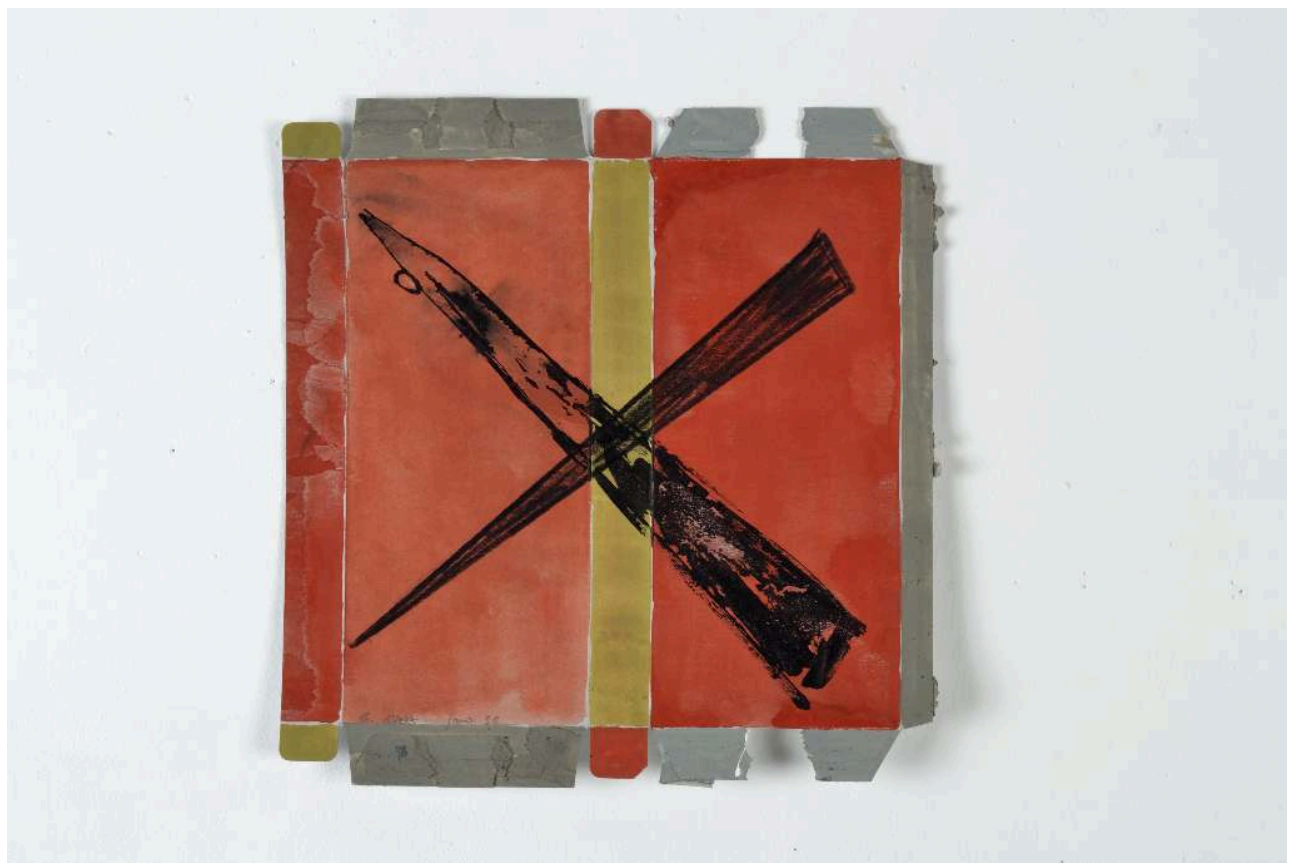

27,1 × 26 cm, acrylique et encre sur emballage chocolat Côte d'Or blanchi au dos, janvier 1996

Photo Hess

Le Raum n'est pas un état de la perception. La cécité est totale et ne peut être considérée comme une étape nécessaire, mais temporaire, au geste du dessin. Ce n'est pas cet aveuglement au motif que suppose Derrida ${ }^{22}$. En effet, à le suivre, le dessinateur ne peut regarder en même temps le motif et la main qui reporte le tracé sur le papier. Tout dessinateur est donc aussi un aveugle, ce que confirmeraient les autoportraits «en aveugle ».

Ce n'est pas non plus le Rien qu'évoque Lyotard à propos de Sam Francis ${ }^{23}$, c'est-à-dire au fond, l'Être, le Visuel, l'Invisible. À propos de Blue Center (1953), il écrit ceci :

Il avait regardé la lumière sur l'océan, de sa chambre d'hôpital à San Francisco, entre mort et vie. Il ne regardait pas les effets de lumière mais la matière lumière, the substance of which the light is made. Or il trouve que la substance est noire. L'œil absolu soupçonne qu'il y a une nuit molle au cœur de la parade chromatique qui ravit le regard. A piece of bread with its hard crust and soft inside. ${ }^{24}$

Et plus loin : «Ses yeux [de Sam Francis] émerveillés seront toujours au rendez-vous avec la cécité. Toute son œuvre aura lutté pour la mise en absence du visible mais en accréditant sa présence. La magnificence donne une leçon de ténèbres. $»^{25}$

À propos de Meaningless Gesture (1958) : «Il s'agit toujours de ne pas succomber à la tentation du visible et d'honorer en lui l'obscur pouvoir qui le rend possible. [...] Le geste de peindre in-signifie la trop significative couleur. ${ }^{26}$

En fait, Lyotard n'incorpore-t-il pas ainsi, dans une esthétique négative (comme il y a une théologie négative), ou plutôt une cosmétique de la révélation, une partie des "modernes", selon une condition, la division entre le visible et sa condition dont il faut faire le deuil ? C'est cette différence avec un arrière-monde qui ne serait plus constitutive chez les artistes « contemporains »:

La plainte du Vendredi Saint se propage de verset en verset, la leçon de ténèbres s'épelle lettre après lettre. Une à une, les couleurs rappelleront au regard, par leur 
abondance même, le rien qu'elles lui font oublier. Gris de Paul Klee, noir et blanc de Malevitch. Arakawa dénomme blank l'absence de toute vision. Duchamp juge futile de la baptiser, les couleurs n'existent pas plus que le corps et les yeux, elles ne sont que leurs noms, le déluge est déjà là, on ne peut plus peindre ni se plaindre. Mais ce n'est pas vrai, dit ici l'amant des splendeurs chromatiques. Le deuil doit être perpétué.... ${ }^{27}$ surtout en l'absence de repères sonores fixes, étant fréquemment manipulé sans pouvoir toucher quoi que ce soit puisqu'il est immobilisé sur son lit de douleur par des sangles. Ce corps ne saurait être touchant/être touché, selon le schéma du chiasme cher à Merleau-Ponty. Ce corps est l'objet de nombreuses intrusions, incompréhensibles, de soins qui sont des humiliations, de déplacements ${ }^{30}$ qui sont des arrachements, d'une douleur inimaginable qui témoigne qu'il revient peu à peu à la vie sans avoir la capacité de concevoir un avant et un après puisqu'il n'a de souvenir ni de l'accident ni d'une vie antérieure. Car l'oubli a triomphé. La remontée vers la lumière n'est donc pas synonyme d'une délivrance, d'une reconquête, d'une résurrection au sens testamentaire, mais d'une offensive de l'ennemi. Bref, la lumière, ce sont les ténèbres où des voix se proposent d'expérimenter de nouvelles techniques chirurgicales. Comme Rœsz le dit aujourd'hui, «à cette époque ", les médecins ne s'adressaient pas aux malades, réduits à l'état de gisants ou d'écorchés. À cela s'ajoute le délire du voisin atteint de crises de delirium tremens. S'il y eut un minimum de conscience sensitive (d'après la mémoire qu'il en a aujourd'hui), celle-ci ne pouvait être intentionnelle au sens de la phénoménologie, n'étant pas de l'ordre d'une visée. Mais elle n'était pas non plus de la sphère de l'appropriation d'une extériorité, ce qui suppose, comme le rappelle Derrida dans l'un de ses derniers séminaires, quelque chose qui fût déjà là, de l'ordre du propre. Au fond, l'appropriation suppose toujours qu'un moi rende propre ce qui ne l'est pas, dans tous les sens du terme. On présuppose le propre du moi, ou de son extension, le corps politique. Et puis, on se demande comment sera traité l'étranger (l'impropre) : sera-t-il reçu selon les lois de l'hospitalité ou celles de la guerre ? Y aura-t-il souillure ou non ?31

Or, le Raum n'est pas, pour le supposé moi, un environnement, sonore puis spatial. Il s'impose avant la différenciation entre intérieur et extérieur et, bien sûr, avant l'édification des frontières du moi propre. C'est donc un en-deçà de l'analyse, ce qu'elle ne peut prendre en considération. C'est alors que la peinture est le seul remède, en 
particulier une peinture dont les couleurs se répandent en instituant leur propre support selon une topologie aléatoire qui demande au visiteur de l'atelier de Rœsz de participer à l'enveloppement-développement, au pliage-dépliage, ce qui ne relève pas de la contemplation esthétique classique. Ces toiles, souvent immenses, sont faites pour qu'on s'y roule à s'en aveugler.

Il faut donc considérer que la Mère des Muses ${ }^{32}$, ce n'est pas Mnémosyne, mais l'Oubli. Et sur fond d'oubli, il y a la frontière, non pas entre le propre et l'impropre, mais celle entre hospitalité et inhospitalité ${ }^{33}$. Le Raum permet de situer une hospitalité inattendue : celle des effets d'optique. Le plus attentif des hospitaliers n'aurait pas imaginé que le filtre des persiennes, permettant que le bleu des gyrophares des ambulances, se transformant en un gris-orangé, suscite une sorte de commande, voire un devoir mimétique. Or, ce n'est pas la couleur qui commande, mais un effet d'optique, c'est-à-dire la répétition d'un clignotement comme une succession de clins d'œil. Héraclite (frag. 30) chante un kosmos éternel, incréé, impérissable : «Ce monde-ci, le monde pour tous, nul des dieux ni des hommes ne l'a fait. Mais il était toujours, est et sera feu éternel, s'allumant en mesure et s'éteignant à mesure ».

\section{BIBLIOGRAPHIE}

Bellour Raymond, Le corps du cinéma. Hypnoses, émotions, animalités, Paris, POL, 2009.

Benjamin Walter, L'œuvre d'art à l'époque de sa reproductibilité technique, Euvres complètes, tome III, Paris, Gallimard, 2000.

Benjamin Walter, Fragments : philosophiques, politiques, critiques, littéraires, Christophe Jouanlanne (trad.), Paris, PUF, 2001.

Benjamin Walter, Expérience et pauvreté, CEuvres complètes, t. II, Paris, Gallimard, 2000.

Benjamin Walter, Enfance berlinoise, Jean Lacoste (trad.), Paris, Les Lettres Nouvelles / Maurice Nadeau, 1988.

Bergson Henri, Matière et mémoire, Paris, Flammarion, 2012.

Coureau Didier, Flux cinématographiques, cinématographie des flux, Paris, L'Harmattan, 2010.

Déotte Jean-Louis, Walter Benjamin et la forme plastique, Paris, L'Harmattan, 2012.

Derrida Jacques, Mémoires d'aveugle. L'autoportrait et autres ruines, Paris, Réunion des musées nationaux, 1990.

Didi-Huberman Georges, L'invention de l'hystérie, Paris, Macula, 2012.

Douglas Mary, De la souillure. Essai sur les notions de pollution et de tabou, Paris, La Découverte, 2001.

Lyotard Jean-François, L'économie libidinale, éditions de Minuit, 1974.

Lyotard Jean-François, Le Différend, Paris, éditions de Minuit, coll. « Critique », 1983.

Lyotard Jean-François, Misère de la philosophie, Paris, Galilée, 2000. 
Lyotard Jean-François, Sam Francis. Leçons de Ténèbres « like the paintings of a blind man », Louvain, Presses universitaires de Louvain, 2010.

Morante Elsa, La Storia, Paris, Gallimard, 2004.

Nancy Jean-Luc, Les Muses, Paris, Galilée, 1994.

Reybrouck David van, Congo. Une histoire, Actes Sud, 2012.

Rœsz Germain, L'éclat rouge, un peu de Colmar, Colmar, éditions Jérôme Do Bentzinger, 2009.

Rœsz Germain, Peintures, 1970-2011, Paris, L'Harmattan / Cour carrée, 2012.

Simondon Gilbert, Du mode d'existence des objets techniques, Paris, Aubier, 2012.

Skopin Denis, « Les disparitions politiques et la photographie ", thèse de philosophie, Paris, [à paraître], 2014.

Virilio Paul, Esthétique de la disparition, éditions Le Livre de Poche, coll. « essais », 1994.

Cuvres de Sam Francis citées dans l'ouvrage de J.-F. Lyotard (2010) :

Opposites, 1950.

Grey Cloud Study, 1950.

Untitled, 1988.

Untitled, 1989.

\section{NOTES}

1. David van Reybrouck, Congo. Une histoire, Actes sud, 2012.

2. Henri Bergson, Matière et mémoire, Paris, Flammarion, 2012.

3. Elsa Morante, La Storia, Paris, Gallimard, 2004, p. 354.

4. Jean-François Lyotard, Le Différend, Paris, éditions de Minuit, coll. « Critique », 1983.

5. Elsa Morante, La Storia, Paris, Gallimard, 2004, p. 354.

6. Walter Benjamin, Expérience et pauvreté, Ceuvres complètes, t. II, Paris, Gallimard, 2000. Et notre commentaire : Jean-Louis Déotte, Walter Benjamin et la forme plastique, Paris, L'Harmattan, 2012.

7. Gilbert Simondon, Du mode d'existence des objets techniques, Paris, Aubier, 2012.

8. Denis Skopin, « Les disparitions politiques et la photographie », thèse de philosophie, Paris, [à paraître] 2014.

9. Raymond Bellour, Le corps du cinéma. Hypnoses, émotions, animalités, Paris, POL, 2009. C'est au contraire l'hypnose qui sert de modèle à la réception cinématographique. « Le parti pris essentiel de ce livre suppose une équivalence entre l'état de cinéma compris comme hypnose légère et la masse des émotions éprouvées au cours de la projection d'un film. Mais plutôt que des émotions conventionnelles, de nature psychologique, il s'agit des émotions premières que Daniel Stern a nommées des affects de vitalité : les réactions sensibles induites chez le tout petit enfant par la construction corporelle et psychique de son expérience, qui sont autant de signes précurseurs du style dans l'art. De ces émotions sans nom, aussi variables que toujours recommencées, le cinéma semble par excellence être le lieu, lui qui se donne, dans ses films authentiques, pour la réalité faite art. » (Citation de l'éditeur). On voit que Bellour est à la fois très proche des intuitions de Benjamin-Kracauer, mais en faisant l'économie de la théorie du choc esthétique, pourtant au cœur de L'œuvre d'art à l'époque de sa reproductibilité technique de Benjamin. 
10. Georges Didi-Huberman, L'invention de l'hystérie, Paris, Macula, 2012. En mettant l'accent sur la fabrication photographique de l'hystérie, Didi-Huberman met en place ce qui deviendra la théorie de la survivance des formes.

11. Didier Coureau, Flux cinématographiques, cinématographie des flux, Paris, L'Harmattan, 2010.

12. Germain Rœsz, à propos de la Mélancolie de Lucas Cranach l'Ancien, récemment acquise par le musée Unterlinden : « Les œuvres nourrissent les artistes avec force. La mélancolie s'est insinuée en moi non pas par les quatre humeurs, la bile noire, le flegme, la bile jaune et le sang mais plutôt par le mot de Hugo "La mélancolie, c'est le bonheur d'être triste." S'adonner à la rêverie, regarder le ciel et imaginer Saturne en soi ", Rœsz Germain, L'éclat rouge, un peu de Colmar, Colmar, éditions Jérôme Do Bentzinger, 2009, p. 59.

13. Germain Rœsz, Peintures, 1970-2011, Paris, L'Harmattan / Cour carrée, 2012, p. 143.

14. Paul Virilio, Esthétique de la disparition, éditions Le Livre de Poche, coll. « essais », 1994.

15. Germain Rœsz, Peintures, 1970-2011, Paris, L'Harmattan / Cour carrée, 2012.

16. Walter Benjamin, Fragments: philosophiques, politiques, critiques, littéraires, Christophe Jouanlanne (trad.), Paris, PUF, 2001.

17. Germain Rœsz, L'éclat rouge, un peu de Colmar, Colmar, éditions Jérôme Do Bentzinger, 2009.

18. Ibid., p. 83.

19. Ils sont sur la même bande, celle que décrit Lyotard dans L'économie libidinale, et qu'étudie A. Heathcote, dans le numéro 10 de la revue Appareil, automne 2012, http://appareil.revues.org/ 1507.

20. Walter Benjamin, Enfance berlinoise, Jean Lacoste (trad.), Paris, Les Lettres Nouvelles / Maurice Nadeau, 1988.

21. Jean-François Lyotard, Misère de la philosophie, Paris, Galilée, 2000.

22. Jacques Derrida, Mémoires d'aveugle. L'autoportrait et autres ruines, Paris, Réunion des musées nationaux, 1990.

23. Jean-François Lyotard, Sam Francis. Leçons de Ténèbres "like the paintings of a blind man", Louvain, Presses universitaires de Louvain, 2010.

24. Idem, $\$ 4$.

25. Sam Francis, Opposites, 1950, cité dans $§ 5$ de l'ouvrage de J.-F. Lyotard, op. cit., 2010.

26. Jean-François Lyotard, op. cit., 2010, § 6.

27. Sam Francis, Grey Cloud Study, 1950, cité dans § 7 de l'ouvrage de J.-F. Lyotard, op. cit., 2010.

28. Sam Francis, Untitled, 1988, Idem, § 22.

29. Sam Francis, Untitled, 1989, Idem, § 35.

30. Car dans un hôpital, chaque lit est la quasi-propriété d'un service, c'est-à-dire d'un patron.

31. Mary Douglas, De la souillure. Essai sur les notions de pollution et de tabou, Paris, La Découverte, 2001.

32. Jean-Luc Nancy, Les Muses, Paris, Galilée, 1994.

33. Un critère de l'hospitalité : prendre soin de la douleur. Rendre sensibles les hospitaliers au traitement de la douleur en la sortant de la fatalité théologique. Dès lors, on peut être amené à plonger le patient dans un coma artificiel. 


\section{RÉSUMÉS}

Le terme de «création » s'agissant de la production artistique ne nous semble pas approprié. Parce que le modèle est théologique, cette référence n'apparaissant qu'avec le Romantisme. Il est d'autant plus inapproprié à l'ère numérique où l'artiste n'a plus affaire à de la matière au sens propre, mais à du langage. Ce terme n'avait aucun sens pour les Grecs, où la mémoire était la «mère des Muses. »

Cette maternité peut d'ailleurs être remise en cause, si l'on considère les cas de certains peintres comme Sam Francis aux États-Unis, Beuys en ex-RFA ou Germain Rœsz en France. Quelles sont les caractéristiques d'une production qui surgit après un coma? Nous nous intéresserons donc à des artistes dont l'horizon ne fut pas muséal. Mais hospitalier, dans un sens large...

INDEX

Mots-clés : créativité, oubli, environnement

Personnes citées : Sam Francis, Germain Rœsz 